\title{
Répercussions agronomiques de l'épandage d'effluents et déchets de moulins à huile d'olive
}

\author{
Alain MORISOT \& Jean-Paul TOURNIER \\ I.N.R.A., Station d'Agronomie, B.P. 2078, F 06606 Antibes
}

RÉSUMÉ

\begin{abstract}
Les répercussions agronomiques de l'épandage d'effluents (ou margines) de moulins à huile d'olive sont étudiées au moyen de cultures de ray-grass en pots sous serre et de tests d'incubation sur l'azote minéral. L'épandage de $40 \mathrm{~mm}\left(40 \mathrm{l} / \mathrm{m}^{2}\right)$ de margines sur une plantation de ray-grass diminue le rendement moyen de 45 p. 100 par rapport à celui de la culture de référence. La production de matière sèche des ray-grass semés immédiatement après l'épandage (doses de 40 et $80 \mathrm{~mm}$ ) est égale au tiers de la production de référence.

La salinité extrêmement élevée des margines, leur acidité, la présence possible de polyphénols ou d'autres substances toxiques biodégradables et l'absence de fourniture d'azote minéral, peuvent expliquer ces répercussions négatives. Les semis réalisés $45 \mathrm{j}$ après l'épandage montrent que, lorsque les margines ont eu un effet sur la croissance des végétaux, il a toujours été favorable : si dans 5 traitements (doses de 40 à $80 \mathrm{~mm}$ ), l'apport des margines n'a pas significativement augmenté les rendements, dans 5 autres cas (doses de 20 à $80 \mathrm{~mm}$ ), il les a améliorés. Les analyses de sols permettent d'attribuer une partie de cet effet fertilisant à l'enrichissement en potassium échangeable.

Les tests d'incubation révèlent que les margines peuvent provoquer une légère disparition d'azote nitrique, limitée dans ces essais à 0,2 p. 1000 du poids de margines. L'ensemble de ces tests montre que, dans ces conditions expérimentales et aux doses indiquées, on ne peut pas imputer aux margines une inhibition des microflores ammonifiantes et nitrifiantes du sol. La disparition d'azote nitrique serait provoquée soit par dénitrification, soit par réorganisation.
\end{abstract}

Mots clés additionnels : Margines, azote, azote nitrique, potassium, phytotoxicité.

The agronomic effects of land disposal of waste from olive oil crushers were studied. Experimental crops of rye-grass were grown in pots in the greenhouse, and nitrogen mineralization trials were carried out. The input of the equivalent of $40 \mathrm{~mm}\left(40 \mathrm{~V} / \mathrm{m}^{2}\right)$ of waste on an established rye-grass crop, resulted in a $45 \%$ decrease in yield. When the rye-grass was sown immediately after the input of waste (amounts equivalent to 40 and $80 \mathrm{~mm}$ ) the dry matter yield equaled one third of the reference yield. These negative effects could be explained by the very high salinity of these effluents, their acidity, the possible presence of polyphenols or other toxic biodegradable substances and a lack of nitrate nitrogen. Rye-grass sown 45 days after the input of waste showed positive effects (significant for 5 out of 10 crops). Soil analyses revealed an increase in the amount of exchangeable potassium. Nitrogen mineralization trials revealed a slight decrease of soil nitrate nitrogen, limited to $0,2 \mathrm{mg} \mathrm{N} / \mathrm{g}$ waste. Neither ammonification, nor nitrification inhibition could be ascribed to the waste from the olive oil crusher, within the conditions and amounts studied. So, the disappearance of nitrate nitrogen could be attributed to denitrification or reorganization.

Additional key words : Nitrogen, nitrate nitrogen, potassium, phytotoxicity.

\section{INTRODUCTION}

Les moulins à huile d'olive français ont dû trouver une solution satisfaisante pour l'élimination de leurs effluents, appelés "margines». La figure 1 montre que ces dernières correspondent à l'eau de constitution des olives faiblement diluée par l'eau de rinçage (dans le système par pression discontinue qui est encore presque général en France). Or, on ne disposait pas de station d'épuration adaptée (DELLA MONICA et al.,
1978). Au contraire, il semble que le rejet de margines dans une station d'épuration municipale en perturbe gravement le fonctionnement.

D'autres traitements ont été proposés : la fabrication de protéines par des levures (FIESTAS ROS DE URSINOS, 1966) ou la culture de bactéries et de champignons capables d'utiliser les polysaccharides et les composés ligno-cellulosiques (FIESTAS ROS DE URSINOS, 1979 ; MAUGENET comm. pers., 1979). Des recherches sont également poursuivies sur la digestion 


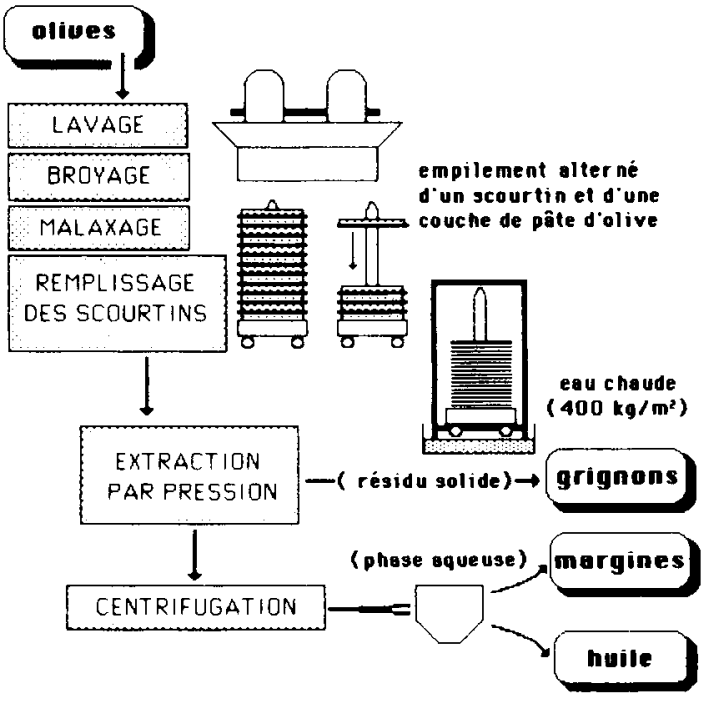

Figure 1

Schéma du fonctionnement d'un moulin à huile d'olive.

Schematic diagram of an olive oil crusher.

anaérobie avec formation de méthane (MAUGENET comm. pers., 1979) ou de composés intermédiaires (FIESTAS ROS DE URSINOS, 1979). On peut même obtenir un effluent avec une DBO réduite à $120 \mathrm{mg} / \mathrm{l}$ en ajoutant une évolution aérobie (FIESTAS ROS DE URSINOS et al., 1979). VITAGLIANO et al. (1975) et DELla MONICA et al. (1978) proposent une concentration à chaud. Enfin, l'oxydation physico-chimique, l'osmose inverse (POMPEI \& CODOvilli, 1974), l'ultrafiltration, l'électrodialyse sont également envisagées (FIESTAS ROS DE URSINOS, 1979). Toutefois, chacun de ces traitements implique un investissement financier difficile a assumer pour la plupart des monlins francais. Une solution moins coúteuse est l'ćvaporation en bassin. D'après ALvAREZ MARTINEZ (1979), elle donne satisfaction et est maintenant très répandue dans l'Espagne du Sud.

La solution très originale étudiée par FIORINO \& Vitagliano (1968) et Vitagliano (1969) ne semble pas être passée dans la pratique. Il s'agissait d'asperger les branches d'oliviers avec des margines pour faciliter l'abscission des fruits. ZUCCONI \& BUKOvAC (1969) ont noté le même effet sur cerise acide (Montmorency) et tomate.

Il reste enfin à examiner l'utilisation par l'agriculture. L'épandage sur les terrains cultivés est déconseillé par certains auteurs (POMPEI \& CODOvilLI, 1974 ; IELMINI et al., 1976) qui invoquent la très forte charge polluante des margines. ZUCCONI \& BUKOVAC (1969) ont mis en évidence l'inhibition de la germination ou de l'élongation des radicelles et hypocotyles de concombre par les margines, même diluées 2 fois. Ils attribuent à la pression osmotique élevée une grande part de cet effet négatif.

La discussion est ouverte. En effet, Alvarez MARTINEZ (1979) a constaté dans la province de Ciudad Real la fréquence des épandages sur jachères ou en fumure de fond après mélange avec des pailles ou des marcs de raisin. Des expériences réussies d'utilisation de margines neutralisées à la chaux sur blé, maïs et olivier sont citées en Espagne (ROMERO \& FIESTAS RoS DE URSinOS, 1960 ; Fiestas Ros de URSINOS,
1977). LlaMAS (comm. pers., 1978) rapporte que l'irrigation d'oliviers ou d'autres cultures avec des margines diluées est courante dans la province de Jaen. On notera cependant les précautions prises lors de ces épandages : rejet sur des jachères, neutralisation et dilution des margines. Des travaux expérimentaux ont été engagés en Italie où DELla MONICA et al. (1978, 1979) étudient l'évolution des margines dans le sol sous des conditions très spéciales : apport extrêmement élevé de $480 \mathrm{~mm}$, sur un sol calcaire, organique et très riche.

On trouvera ici les résultats d'expérimentations effectuées à Antibes, selon la méthodologie utilisée pour des déchets de distilleries vinicoles (MORISOT, 1986). D'autres observations avaient déjà été publiées (MORISOT, 1979).

\section{MATÉRIEL ET MÉTHODE}

Le produit étudié est l'effluent des moulins à huile d'olives, appelé "margines" (fig. 1). Des prélèvements ont été effectués dans 3 moulins. On en trouvera la composition au tableau 1. Ces margines sont acides, leur salinité globale est très élevée et elles contiennent plusieurs $\mathrm{g} / \mathrm{l}$ de potassium. Par contre, elles semblent ne renfermer ni sodium, ni azote minéral (dans les conditions de l'examen).

Les cultures expérimentales de ray-grass en pots et les tests d'incubation pour l'azote ont été effectués selon la méthodologie exposée dans une autre publication (MORISOT, 1986). Le tableau 1 précise la nature des terres utilisées et le tableau 2 décrit les 5 situations expérimentales étudiées. I es répétitions sont an nombre de 4. I. tahleau 3 indique les apporis d'éléments fortilisants, en $\mathrm{mg}$ /pot, effectućs par les margines.

Par la suite, on verra que les premiers tests d'incubation réalisćs montrent que l'addition de margines (qui pourtant contiennent de l'azote organique) à un sol ne provoque pas une fourniture d'azote minéral. Cela peut être dû à la lenteur de la minéralisation de cet azote des margines mais aussi à un effet inhibiteur des effluents sur la microflore du sol. Pour tenter de trancher entre ces 2 hypothèses, on a mélangé dans une même série d'échantillons des margines et des vinasses de betteraves qui fournissent rapidement de l'azote nitrique quand elles sont utilisées seules.

\section{RÉSULTATS}

\section{A. Cultures expérimentales}

\section{Epandage pendant la culture}

L'épandage de $40 \mathrm{~mm}\left(40 \mathrm{l} / \mathrm{m}^{2}\right)$ de margines sur une plantation de ray-grass au début d'un cycle de repousse des parties aériennes a provoqué une baisse significative du rendement (test de NEWMAN à 5 p. 100) de la coupe suivante. Le rendement moyen est alors diminué de 45 p. 100 par rapport à celui des cultures de référence (tabl. 4). 
TABLEAU 1

Analyse des terres et des margines utilisées.

Composition of soil and wastes.

Analyse des terres

\begin{tabular}{|c|c|c|c|c|c|c|c|c|c|c|}
\hline$\underset{0 \%}{\text { Argile }}$ & $\begin{array}{l}\mathrm{pH} \\
\text { eaut }\end{array}$ & $\begin{array}{l}\mathrm{pH} \\
\mathrm{KCl}\end{array}$ & $\begin{array}{c}\text { Calcaire } \\
\text { actif }\end{array}$ & $\begin{array}{c}\mathrm{C} \\
\text { total }\end{array}$ & $\stackrel{N}{\text { total }}$ & $\begin{array}{l}\mathrm{P}_{2} \mathrm{O}_{5} \\
\text { Joret }\end{array}$ & $\begin{array}{l}\mathrm{K}_{2} \mathrm{O} \\
\text { cch. }\end{array}$ & $\begin{array}{l}\text { Ca } \\
\text { ech. }\end{array}$ & $\begin{array}{l}\mathrm{Mg} \\
\text { ech. }\end{array}$ & $\begin{array}{l}\text { CEC } \\
\text { meq/ } \\
100 \mathrm{~g}\end{array}$ \\
\hline
\end{tabular}

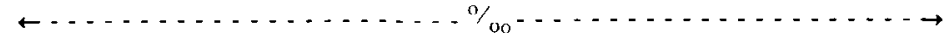

Terre $n^{\circ} 1$

argilo-limoneuse peu calcaire
Terre $n^{\circ} 2$

$\begin{array}{llllllllllll}35 & 8,2 & 7,1 & 18 & 9,6 & 0,87 & 0,16 & 0,34 & 7,82 & 0,81 & 0,05 & 29,6\end{array}$

argilcuse peu calcaire

Terre $\mathrm{n}^{\circ} 3$

$\begin{array}{llllllllllll}33 & 7,9 & 6,8 & 10 & 7,6 & 1,02 & 0,08 & 0,35 & 3,22 & 0,57 & 0,07 & 17,4\end{array}$

sableuse calcaire

Terre $\mathrm{n}^{\circ} 4$

$10 \quad 8,4 \quad 7,5 \quad 60$

$2,4 \quad 0,28 \quad 0,05$

$0,02 \quad 6,58$

$0,13 \quad 0,06$

6,6

limono-argileuse calcaire

$\begin{array}{llll}26 & 8,0 & 7,2 & 30\end{array}$

$13,5 \quad 1,48$

$0,11 \quad 0,21$

$0,32 \quad 0,02 \quad 12,6$

Analyse des margines

\begin{tabular}{|c|c|c|c|c|c|c|c|c|c|c|c|}
\hline & $N$ & $P$ & K & $\mathrm{Mg}$ & $\mathrm{Ca}$ & $\mathrm{Na}$ & $\mathrm{N}-\mathrm{NO}_{3}$ & $\mathrm{~N}-\mathrm{NH}_{4}$ & MS & $\begin{array}{l}\mathrm{CE} \\
\mathrm{mS}\end{array}$ & $\mathrm{pH}$ \\
\hline & & & & & & & & & MF & & \\
\hline Moulin 1 & 0,60 & 0,29 & 2,93 & 0,08 & 2,70 & traces & $<5$ & $<5$ & 6,8 & 8,2 & 4,7 \\
\hline Moulin 2 & & & & & & & & & & & \\
\hline prélèvement 1 & 0,62 & 0,36 & 4,43 & 0,15 & 6,60 & traces & $<5$ & $<5$ & 5,6 & 9,5 & 5,0 \\
\hline rélèvement 2 & 0,64 & 0,86 & 9,30 & 0,28 & 10,80 & traces & $<5$ & $<5$ & 7,5 & - & - \\
\hline prélèvement 3 & & & & & & & & & & 16,0 & 5,1 \\
\hline prélèvement 4 & & & & & & & & & & 16,0 & 5,1 \\
\hline Moulin 3 & 0,52 & 0,40 & 5,75 & 0,14 & 8,20 & traces & & & 5,8 & 11,4 & 5,4 \\
\hline
\end{tabular}

TABLEAU 2

Description des conditions expérimentales de culture.

Description of experimental crops.

\begin{tabular}{|c|c|c|c|c|c|c|c|}
\hline $\begin{array}{c}\text { Essai relaté } \\
\text { en }\end{array}$ & Culture & Terre & $\begin{array}{l}\text { Doses de } \\
\text { margines }\end{array}$ & Apport & $\begin{array}{c}\text { Date } \\
\text { d'épandage }\end{array}$ & $\begin{array}{c}\text { Date } \\
\text { de semis }\end{array}$ & $\begin{array}{l}\text { Date } \\
\text { de récolte }\end{array}$ \\
\hline III Al & $\begin{array}{l}\text { ray grass } \\
\text { en pots }\end{array}$ & $\begin{array}{c}\mathrm{n}^{\circ} 1 \\
800 \mathrm{~g} / \text { pot }\end{array}$ & $\begin{array}{c}40 \mathrm{~mm} \\
100 \mathrm{ml} / \mathrm{pot}\end{array}$ & $\begin{array}{l}\text { arrosage des } \\
\text { pots et } \\
\text { recyclage }\end{array}$ & $13 / 1$ & $\begin{array}{l}\text { coupe } \\
\text { le } 12 / 1\end{array}$ & $24 / 2$ \\
\hline III A2 & $\gg$ & $\gg$ & 40 et $80 \mathrm{~mm}$ & $\gg$ & $\gg$ & $\gg$ & $\gg$ \\
\hline III A3 & $\gg$ & $\mathrm{n}^{\circ} 2: 1100 \mathrm{~g}$ & $20 \mathrm{~mm}$ & $\ll$ & $12 / 4$ & $27 / 4$ & $23 / 5$ \\
\hline et A4 & & $\begin{array}{c}\mathrm{n}^{\circ} 3: 1250 \mathrm{~g} \\
\text { par pot }\end{array}$ & $60 \mathrm{ml} /$ pot & & $»$ & $26 / 5$ & $25 / 6$ \\
\hline III A4 & $\gg$ & $\begin{array}{c}\mathrm{n}^{\circ} 1 \\
800 \mathrm{~g} / \mathrm{pot}\end{array}$ & $\begin{array}{c}40 \text { et } 80 \mathrm{~mm} \\
100 \text { et } 200 \mathrm{ml} / \text { pot }\end{array}$ & $\gg$ & $13 / 1$ & $2 / 3$ & $31 / 3$ \\
\hline III A5 & $\begin{array}{l}\text { ray-grass } \\
\text { en pots }\end{array}$ & $\begin{array}{c}\mathrm{n}^{\circ} 4 \\
1 \mathrm{I} 00 \mathrm{~g} / \mathrm{pot} \\
\quad \text { remp }\end{array}$ & $\begin{array}{l}10 \text { et } 20 \mathrm{~mm} \\
\text { is avec la terre prél }\end{array}$ & $\begin{array}{c}" y \\
\text { ée dans un es }\end{array}$ & $25 / 3$ & $28 / 8$ & $28 / 9$ \\
\hline
\end{tabular}

TABLEAU 3

Apports d'éléments fertilisants par les margines (mg/pot).

Inputs of fertilising elements due to wastes ( $\mathrm{mg} / \mathrm{pot}$ ).

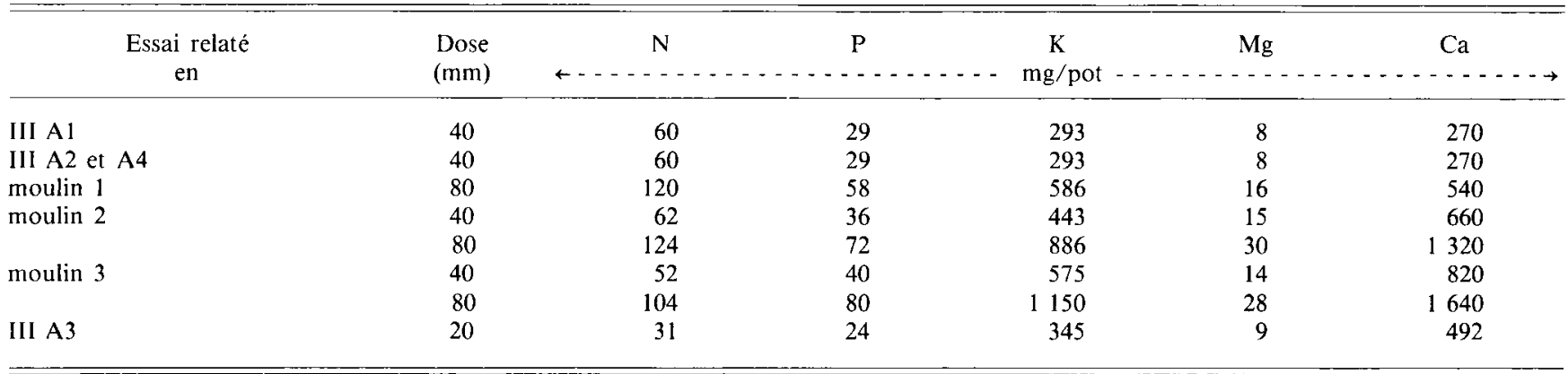


TABLEAU 4

Production des parties aériennes de ray-grass ( $g$ de matière sèche/pot). Yields of rye-grass leaves ( $g$ dry matter per pot).

\begin{tabular}{|c|c|c|c|c|c|}
\hline $\begin{array}{c}\text { Fssai relaté } \\
\text { en III A }\end{array}$ & Traitements & Moyenne & $\mathrm{F}$ & $\begin{array}{l}\text { Degré de liberté } \\
\text { traitements }\end{array}$ & Erreur \\
\hline $\begin{array}{c}1 \\
\text { épandage pendant } \\
\text { la culture }\end{array}$ & $\begin{array}{l}\text { terre seule } \\
+40 \mathrm{~mm} \text { margines }\end{array}$ & $\begin{array}{l}0,93 \mathrm{a} \\
0,49 \mathrm{~b}\end{array}$ & $5,3^{*}$ & 2 & 22 \\
\hline $\begin{array}{l}2 \\
\text { semis immédiat } \\
\text { après l'épandage }\end{array}$ & $\begin{array}{l}\text { terre seule } \\
+40 \mathrm{~mm} \text { margines } 1 \\
+80 \mathrm{~mm} " \\
+40 \mathrm{~mm} \text { margines } 2 \\
+80 \mathrm{~mm} 》 \\
+40 \mathrm{~mm} \text { margines } 3 \\
+80 \mathrm{~mm} \gg\end{array}$ & $\begin{array}{ll}0,47 & \mathrm{~A} \\
0,14 \mathrm{~B} \\
0,12 \mathrm{~B} \\
0,15 \mathrm{~B} \\
0,14 \mathrm{~B} \\
0,17 \mathrm{~B} \\
0,13 \mathrm{~B}\end{array}$ & $119^{* *}$ & 6 & 18 \\
\hline $\begin{array}{c}3 \\
\text { semis } 15 \text { jours } \\
\text { après l'épandange }\end{array}$ & $\begin{array}{l}\text { terre } \mathrm{n}^{\circ} 2 \text { (argileuse) } \\
+20 \mathrm{~mm} \text { margines } \\
\text { terre } \mathrm{n}^{\circ} 3 \text { (sableuse) } \\
+20 \mathrm{~mm} \text { margines }\end{array}$ & $\begin{array}{l}0,80 \mathrm{~A} \\
0,60 \mathrm{~B} \\
0,38 \mathrm{C} \\
0,38 \mathrm{C}\end{array}$ & $30^{* *}$ & 3 & 9 \\
\hline $\begin{array}{c}4 \\
\text { semis } 44 \text { jours } \\
\text { après l'épandage }\end{array}$ & $\begin{array}{l}\text { terre } n^{\circ} 2 \text { (argileuse) } \\
+ \text { fertilisation } \\
+20 \mathrm{~mm} \text { margines } \\
\text { terre } n^{\circ} 3 \text { (sableuse) } \\
+ \text { fertilisation } \\
+20 \mathrm{~mm} \text { margines }\end{array}$ & $\begin{array}{l}0,90 \mathrm{~B} \\
3,10 \mathrm{~A} \\
0,83 \mathrm{~B} \\
0,65 \mathrm{~B} \\
3,40 \mathrm{~A} \\
0,59 \mathrm{~B}\end{array}$ & $107^{* *}$ & 5 & 18 \\
\hline $\begin{array}{c}4 \\
\text { semis } 48 \text { jours } \\
\text { après l'épandage }\end{array}$ & $\begin{array}{l}\text { terre seule } \\
+40 \mathrm{~mm} \text { margines } 1 \\
+80 \mathrm{~mm} " \\
+40 \mathrm{~mm} \text { margines } 2 \\
+80 \mathrm{~mm} " \\
+40 \mathrm{~mm} \text { margines } 3 \\
+80 \mathrm{~mm} \gg\end{array}$ & $\begin{array}{l}0,24 \mathrm{~B} \\
0,26 \mathrm{~B} \\
0,24 \mathrm{~B} \\
0,37 \mathrm{AB} \\
0,43 \mathrm{~A} \\
0,31 \mathrm{AB} \\
0,27 \mathrm{~B}\end{array}$ & $6,5^{* *}$ & 6 & 18 \\
\hline
\end{tabular}

Sur un même essai, les moyennes associées à une même lettre ne sont pas distinctes pour un test de NEwMAN (minuscules $=$ niveau $5 \%$; majuscules $=$ niveau $1 \%$ ).

Les valeurs de F peuvent être différentes de 1 au niveau $5 \%\left(^{*}\right)$ ou $1 \%(* *)$.

\section{Semis immédiatement après l'épandage}

La production de matière sèche en $42 \mathrm{j}$ par les raygrass semés immédiatement après l'épandage des margines est très réduite par rapport à celle de la culture de référence. Cette diminution est significative au niveau de 1 p. 100 pour un test de Newman. On ne décèle aucune différence entre les 3 margines utilisées, ni entre les 2 doses ( 40 et $80 \mathrm{~mm}$ ).

\section{Semis $15 j$ après l'épandage}

Deux terres différentes ont été utilisées pour cet essai. Avec la terre argileuse, la production de matière sèche à $26 \mathrm{j}$ a été significativement diminuée par l'apport de margines (test de NEWMAN au niveau 1 p. 100). Par contre, sur la terre sableuse, cette production est identique avec et sans épandage de margines.

\section{Semis $45 j$ après l'épandage}

La récolte effectuée environ $75 \mathrm{j}$ après l'épandage révèle l'effet favorable de 3 épandages de margines : margine du moulin 2 aux 2 doses $(40$ et $80 \mathrm{~mm}$ ) et margine du moulin 3 à la dose de $40 \mathrm{~mm}$. Dans toutes les autres situations, l'épandage ne provoque aucune différence de rendement avec la culture témoin sur terre seule et sans fertilisation (test de NEWMAN, niveau 5 p. 100).

\section{Semis 5 mois après l'épandage}

Une coupe a été effectuée $30 \mathrm{j}$ après ce semis sur des échantillons de terre prélevés dans l'horizon 0$10 \mathrm{~cm}$ d'un terrain d'épandage. L'analyse des productions de matière sèche montre que les terres correspondant aux 2 doses 20 et $60 \mathrm{~mm}$ de margines ont des effets identiques et significativement favorables (test de NEWMAN au niveau 1 p. 100).

\section{B. Evolution physico-chimique des sols}

Les terres utilisées dans les essais rapportés cidessus (cf. tabl. 2 et chap. III.A 2, 3, 4) ont été analysées 2 mois et demi après leur mélange avec les margines (tabl. 5).

Sur les 2 terres où la dose de margines a été de $20 \mathrm{~mm}, 2$ paramètres seulement ont été modifiés par l'épandage. La teneur en potassium échangeable a ét augmentée de 0,16 p. $1000 \mathrm{~K}_{2} \mathrm{O}$ (terre sableuse) ou 0,25 p. $1000 \mathrm{~K}_{2} \mathrm{O}$ (terre argileuse). La salinité réelle a légèrement progressé, mais elle reste tout à fait acceptable. 
TABLEAU 5

Evolution physico-chimique des terres après épandage. Soil analyses with and without added waste.

\begin{tabular}{|c|c|c|c|c|c|c|c|c|}
\hline & \multicolumn{4}{|c|}{$\begin{array}{l}\text { Terre } n^{\circ} 1 \\
\text { argilo-limoneuse }\end{array}$} & \multicolumn{2}{|c|}{$\begin{array}{l}\text { Terre } n^{\circ} 2 \\
\text { argileuse }\end{array}$} & \multicolumn{2}{|c|}{$\begin{array}{c}\text { Terre } n^{\circ} 3 \\
\text { sableuse }\end{array}$} \\
\hline & témoin & & $\begin{array}{l}\mathrm{mm} \mathrm{m} \\
\mathrm{u} \mathrm{mou}\end{array}$ & & témoin & $\begin{array}{l}+20 \mathrm{~mm} \\
\text { margines }\end{array}$ & témoin & $\begin{array}{l}+20 \mathrm{~mm} \\
\text { margines }\end{array}$ \\
\hline & & $n^{\circ} 1$ & $\mathrm{n}^{\circ} 2$ & $\mathrm{n}^{\circ} 3$ & & & & \\
\hline pH eau & 8,2 & 8,2 & 8,1 & 8,1 & 7,9 & 7,9 & 8,4 & 8,4 \\
\hline $\mathrm{pH} \mathrm{KCl}$ & 7,1 & 7,2 & 7,2 & 7,2 & 6,8 & 7,1 & 7,5 & 7,6 \\
\hline $\mathrm{CEC}(\mathrm{me} / 100 \mathrm{~g})$ & 29,6 & 30,6 & 29,9 & 29,4 & 17,4 & 16,9 & 6,6 & 6,9 \\
\hline $\mathrm{P}_{2} \mathrm{O}_{5}$ Joret-Hebert $\%$ & 0,16 & 0,22 & 0,20 & 0,23 & 0,08 & 0,08 & 0,05 & 0,06 \\
\hline $\mathrm{K}_{2} \mathrm{O}$ & 0,34 & 1,21 & 1,18 & 1,46 & 0,35 & 0,60 & 0,02 & 0,18 \\
\hline $\mathrm{Ca}$ & 7,82 & 7,54 & 7,26 & 7,18 & 3,22 & 3,04 & 6,58 & 6,35 \\
\hline $\mathrm{Mg}$ & 0,81 & 0,88 & 0,84 & 0,83 & 0,57 & 0,53 & 0,13 & 0,14 \\
\hline $\mathrm{Na} \quad \#$ & 0,05 & 0,06 & 0,06 & 0,06 & 0,07 & 0,07 & 0,06 & 0,06 \\
\hline Salinité réelle $(\%)$ & & & & & 0,00 & 0,20 & 0,24 & 0,36 \\
\hline
\end{tabular}

L'évolution de la terre argilo-limoneuse arrosée avec $80 \mathrm{~mm}$ de margines est beaucoup plus marquée : le potassium échangeable a augmenté en moyenne de 0,95 p. $1000\left(\mathrm{~K}_{2} \mathrm{O}\right)$, l'azote total de 0,12 p. 1000 , le phosphore assimilable (JORET-HÉBERT) de $0,06 \mathrm{p}$. $1000\left(\mathrm{P}_{2} \mathrm{O}_{5}\right)$.

\section{Tests d'incubation sur l'azote minéral}

Les résultats de ces tests sont rassemblés dans la figure 2. La minéralisation de la terre seule fournit $30 \mathrm{ppm}$ environ d'azote nitrique en $16 \mathrm{j}$. L'évolution du mélange terre +5 p. 100 de margines est très différente : la teneur en $\mathrm{N}$ nitrique passe de 7 à $0 \mathrm{ppm}$ pendant les 5 premiers jours et est encore nulle au $13^{\mathrm{e}} \mathrm{j}$. La teneur en $\mathrm{N}$ ammoniacal reste toujours nulle.

On a pu confirmer que l'apport de margines pouvait entraîner la disparition de l'azote nitrique ajouté au début du test d'incubation. Selon les échantillons de margines, de 12 à 28 ppm d'azote nitrique ont ainsi disparu en $7 \mathrm{j}$, soit 0,1 à $0,2 \mathrm{p} .1000 \mathrm{du}$ poids de margines apportées. Cependant, comme dans un des traitements il ne restait plus d'azote nitrique disponible, on ne peut être assuré d'avoir atteint le taux de disparition maximum.

On a voulu encore préciser l'influence d'un apport de margines sur la dynamique de l'azote et notamment mettre en évidence une éventuelle inhibition de la minéralisation ou de la nitrification. Dans ce but, on a observé, dans une série de tests d'incubation, l'influence des margines sur l'évolution d'un produit dont l'épandage provoque normalement la formation de grandes quantités d'azote ammoniacal qui nitrifie progressivement par la suite. Ce produit est de la vinasse de betteraves.

L'incubation d'un mélange de terre et de 5 p. 100 de vinasse de betteraves (fig. 2) donne des résultats identiques, que l'on ait ajouté ou non des margines (à la dose de 5 p. 100). Ni l'ammonification, ni la nitrification ne sont modifiées. Dans cette comparaison, on n'observe aucune disparition d'azote nitrique.

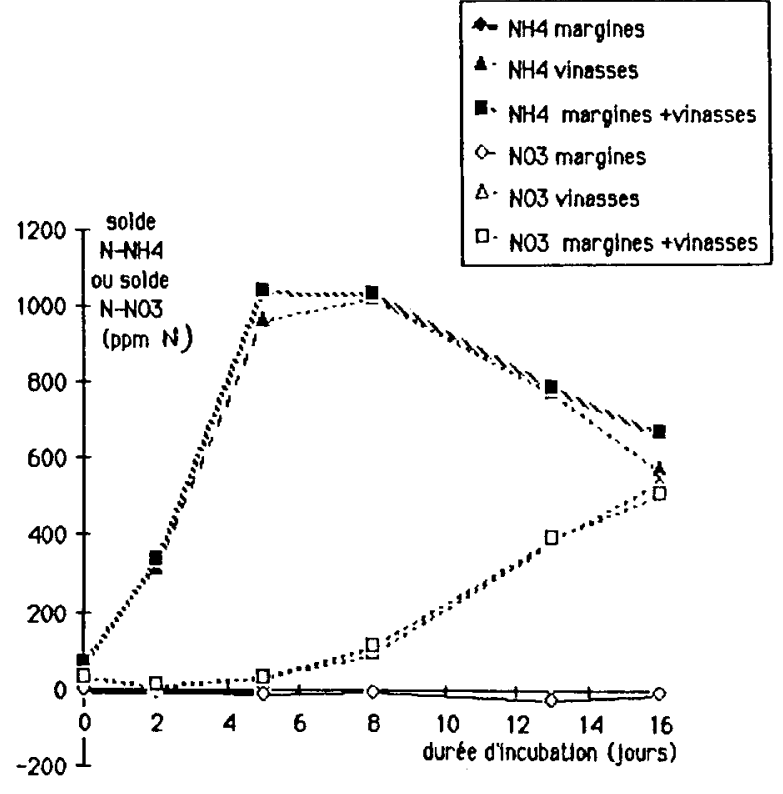

Figure 2

Test de minéralisation de l'azote d'une terre enrichie : de 5 p. 100 de margines,

ou de 5 p. 100 de vinasses de betteraves,

ou du mélange 5 p. 100 de margines et 5 p. 100 de vinasses de betteraves.

Nitrogen mineralisation trial on a sou enriched, with $5 \%$ of olive oil crusher waste, or with $5 \%$ of beet distillery waste. or with a mixture of $5 \%$ of each.

\section{DISCUSSION}

\section{- Pendant 2 semaines après l'épandage}

Les cultures en pots effectuées pendant l'épandage ou immédiatement après ont toutes deux fourni une production de matière sèche très inférieure à celle des cultures de référence. On avait trouvé, dans un essai au champ réalisé par ailleurs, les mêmes répercussions négatives de l'épandage des margines sur des salades repiquées après un délai de $10 \mathrm{j}$ seulement. 
Cet effet est déjà moins marqué pour un semis réalisé $15 \mathrm{j}$ après l'épandage. Avec une des 2 terres ainsi étudiées, le rendement a chuté significativement. Avec l'autre, il n'a pas été modifié par rapport à celui de la référence.

Il semble donc qu'on ait ainsi caractérisé une certaine phytotoxicité des margines vis-à-vis de la culture en place pendant l'épandage ou installée peu de temps après. Pour l'expliquer, on peut citer :

- la salinité extrêmement élevée des margines el leur réaction très acide,

- les teneurs élevées en polyphénols (MAUGENET comm. pers., 1979). La disparition ultérieure de la phytotoxicité impliquerait alors soit leur dégradation, soit leur inactivation,

- l'absence de fourniture d'azote minéral ou le blocage de cet élément.

Les tests d'incubation révèlent que les margines peuvent provoquer une légère disparition d'azote nitrique, limitée dans ces essais à 0,2 p. 1000 du poids de margines. Par ailleurs, on a pu montrer qu'un apport de margines à la dose de 5 p. 100 (soit approximativement $150 \mathrm{t} / \mathrm{ha}$, mélangées sur $20 \mathrm{~cm}$ de profondeur) ne modifiait en rien les processus de minéralisation, puis de nitrification provoqués par l'épandage d'un autre produit très fermentescible.

L'ensemble de ces observations montre que, dans nos conditions expérimentales et aux doses indiquées, on ne peut pas imputer aux margines une inhibition des microflores ammonifiantes et nitrifiantes du sol. La disparition d'azote nitrique serait provoquée soit par dénitrification, soit par réorganisation. Des expériences ultérieures avec marquage seraient nécessaires pour trancher ce point.

- Par la suite

Quand les margines ont eu un effet sur la croissance des végétaux, il a toujours été favorable. En effet, si dans 5 traitements (doses de 40 à $80 \mathrm{~mm}$ ) l'apport des margines n'a pas significativement augmenté les rendements, dans 5 autres cas (doses de 20 à $80 \mathrm{~mm}$ ), il les a améliorés.

D'après les analyses de sols, on peut attribuer une partie de cet effet fertilisant à l'enrichissement en potassium échangeable qui est toujours associé à l'épandage. Par contre, ce n'est qu'avec les fortes doses $(80 \mathrm{~mm})$ que l'enrichissement en phosphorc assimilable devient mesurable. Enfin, la fourniture d'azote nitrique par les margines semble être nulle ou négligeable même après une incubation de 2 mois.

\section{CONCLUSION}

De ces essais et pour le cas des sols calcaires, on retiendra les conclusions suivantes :

- Les margines apportées à des doses de 10 à $80 \mathrm{~mm}$ sur une culture en végétation ou immédiatement avant le semis ont un effet défavorable sur la production. Par contre, le test d'incubation sur l'azote minéral prouve l'absence d'effet inhibiteur sur la microflore du sol concernée.

- Quand un délai d'au moins 2 semaines (dans les conditions expérimentales utilisées) s'est écoulé entre l'épandage et le semis, l'apport de margines ne modifie pas la croissance de la végétation ou l'accroît légèrement. Cette éventuelle valeur fertilisante pourrait provenir de l'apport de potassium et à un moindre degré de phosphore. La fourniture d'azote nitrique semble être tout à fait négligeable dans la période étudiée ( 2 mois et demi).

Recu le 17 mars 1985. Accepté le 23 octobre 1985.

RÉFÉRENCES BIBLIOGRAPHIQUES

Alvarez Martinez A., 1979. Actuaciones realizadas por los almazareros para la eliminacion de los vertidos de alpechin a cauces publicos. XV Asamblea de miembros del Inst. de la Grasa y sus derivados, Sevilla, Mayo, 1979, 6 p.

Della Monica M., Potenz D., Volpicella M., 1978. Effetto inquinante delle acque reflue della lavorazione delle olive su terreno agrario. I : Evoluzione del $\mathrm{pH}$, dei composti azotati e dei fosfati. Inquinamento, $20(10), 81-87$

Della Monica M., Potenz D., Volpicella M., 1979. Effetto inquinante delle acque reflue della lavorazione delle olive su terreno agrario. II : Evoluzione dei lipidi, dei polifenoli e delle sostanze organiche in generale. Inquinamento, 21 (1), 27-30.

Fiestas Ros de Ursinos J. A., 1966. Estudio del alpechin para su aprovechamiento industrial. VII : Instalacion comercial para la obtencion de levaduras pienso. Grasas y Aceites, 17 (2), 41-47.

Fiestas Ros de Ursinos J. A., 1977. Depuracion de aguas residuales en las industrias de aceitunas y aceites de oliva. Grasas y Aceites, 28 (2), 113-121.

Fiestas Ros de Ursinos J. A., 1979. Informacion sobre tratamiento de aguas residuales en las industrias de grasas. $X V$ Asamblea de miembros del Inst. de la Grasa y sus derivados, Sevilla, Mayo, $1979,19 \mathrm{p}$.

Fiestas Ros de Ursinos J. A., Janer del Valle M. L., Leon Cabello R., Navarro Gamero R., 1979. Depuracion anaerobia-aerobia del alpechin. $X V$ Asamblea de miembros del Inst. de la Grasa y sus derivados, Sevilla, Mayo, 1979, 12 p.

Fiorino P., Vitagliano C., 1968. Impiego di prodotti chimici atti a facilitare la raccolta delle olive. Ricerca sull'efficacia delle « acque di vegetazione ». L'Italia Agricola, 105 (10), 7 p.

Ielmini M., Sanna M., Pelosi N., 1976. Indagine sulle acque di rifiuto degli stabilimenti di produzione olearia in provincia di Roma : possibilita di depurazione. Industrie alimentari, 15, 11 (133), 123-131.

Morisot A., 1979. Utilisation des margines par épandage. L'Olivier, 19 (1), 8-13.

Morisot A., 1986. Utilisation agricole de quelques déchets des distilleries de vins rouges. Agronomie, 6 (2).

Pompei C., Codovilli F., 1974. Risultati preliminari sul trattamento di depurazione delle acque di vegetazione delle olive per osmosi inversa. Scienza e technologia degli alimenti, 4, 363-364. 
Romero A. A., Fiestas Ros de Ursinos J. A., 1960. Estudio del alpechin para su aprovechamiento industrial. Ensayos efectuados para su posible utilisacion como fertilizante. Grasas y Aceites, 11 (3), 123-124.

Vitagliano C., 1969. Ricerce sull'impiego di prodotti chimici atti a facilitare la raccolta delle olive. X. Ricerche comparative sull'efficacia di trattamenti con "olio di oliva " e con acque di vegetazione. Scienza e technica Agraria, IX (2-3), 13 p.
Vitagliano M., Pantaleo V. N., Padula M., 1975. Una possible utilizzazione delle acqua di vegetazione delle olive. Atti del 5 Simposio nazionale sulla conservazione della natura, Bari, 22-27 aprile 1975, Vol. II, 243-254.

Zucconi F., Bukovac M. J., 1969. Analisi sull'attivita biologica delle " acqua di vegetazione " delle olive. Riv. dell'ortoflorofrutticoltura italiana, 53 (5), 443-461. 